In this context, one of the most rapidly growing areas of research in vaccinology is maternal immunization, aimed at transplacental protection via maternal antibodies. The report by Marchant (1) suggests that under appropriate conditions of prenatal stimulation, this protective net can potentially be broadened to include the generation of protective cellular immunity in the neonate. Further research into the underlying mechanism(s) of prenatal $\mathrm{T}$ cell priming in this and other congenital infections may provide important new clues to guide the development of safe vaccines that aim to achieve a comparable end.

1. Marchant, A., et al. 2003. Mature CD8 ${ }^{+}$T lymphocyte response to viral infection during fetal life.J. Clin. Invest. 111:1747-1755. doi:10.1172/JCI200317470.

2. Wegmann, T.G., Lin, H., Guilbert, L., and Mosmann, T.R. 1993. Bidirectional cytokine interactions in the maternal-fetal relationship: is successful pregnancy a Th2 phenomenon? Immunol. Today. 14:353-356
3. Krishnan, L., Guilbert, L.J., Wegmann, T.G., Belosevic, M., and Mosmann, T.R. 1996. T helper 1 response against Leishmania major in pregnant C57BL/ 6 mice increases implantation failure and fetal resorptions. J. Immunol. 156:653-662.

4. Munn, D.H., et al. 1998. Prevention of allogeneic fetal rejection by tryptophan catabolism. Science. 281:1191-1193.

5. Roth, I., et al. 1996. Human placental cytotrophoblasts produce the immunosuppressive cytokine interleukin 10. J. Exp. Med. 184:539-548.

6. Guller, S., and LaChapelle, L. 1999. The role of placental Fas ligand in maintaining immune privilege at maternal-fetal interface. Semin. Reprod. Endocrinol. 17:39-44.

7. Hilkens, C.M., Kalinski, P., de Boer, M., and Kapsenberg, M.L. 1997. Human dendritic cells require exogenous interleukin-12-inducing factors to direct the development of naive T-helper cells toward the Th1 phenotype. Blood. 90:1920-1926.

8. Szekeres-Bartho, J., Faust, Z., Varga, P., Szereday, L., and Kelemen, K. 1996. The immunological pregnancy protective effect of progesterone is manifested via controlling cytokine production. Am. J. Reprod. Immunol. 35:348-351.

9. Ulevitch, R.J. 1999. Endotoxin opens the Tollgates to innate immunity. Nat. Med. 5:144-145.

10. Holt, P.G., and Sly, P.D. 2002. Interactions between RSV infection, asthma, and atopy: unraveling the complexities. J. Exp. Med. 196:1271-1275

11. Rowe, J., et al. 2001. Heterogeneity in diphtheriatetanus-acellular pertussis vaccine-specific cellu- lar immunity during infancy: relationship to variations in the kinetics of postnatal maturation of systemic Th1 function. J. Infect. Dis. 184:80-88.

12. Goriely, S., et al. 2001. Deficient IL-12(p35) gene expression by dendritic cells derived from neonatal monocytes. J. Immunol. 166:2141-2146.

13. Yap, G., Pesin, M., and Sher, A. 2000 . IL-12 is required for the maintenance of IFN- $\gamma$ produc tion in T cells mediating chronic resistance to the intracellular pathogen, Toxoplasma gondii. J. Immunol. 165:628-631.

14. White, G.P., Watt, P.M., Holt, B.J., and Holt, P.G. 2002. Differential patterns of methylation of the IFN $\gamma$ promoter at $\mathrm{CPG}$ and non-CPG sites underlie differences in IFN $\gamma$ gene expression between human neonatal and adult CD45RO- T-cells. J. Immunol. 168:2820-2827.

15. Hassan, J., and Reen, D.J. 2001 Human recent thymic emigrants-identification, expansion and survival characteristics. J. Immunol. 167:1970-1976.

16. Deacock, S.J., et al. 1992. Evidence that umbilical cord blood contains a higher frequency of HLA class II-specific alloreactive $\mathrm{T}$ cells than adult peripheral blood. A limiting dilution analysis. Transplantation. 53:1128-1134.

17. Mbawuike, I.N., et al. 2001. HLA-restricted CD8 cytotoxic T lymphocyte, interferon- $\gamma$, and interleukin-4 responses to respiratory syncytial virus infection in infants and children. J. Infect. Dis. 183:687-696.

18. Hermann, E., et al. 2002. Human fetuses are able to mount an adultlike CD8 T-cell response. Blood. 100:2153-2158.

\title{
New insights into a persistent problem - chlamydial infections
}

\section{Richard P. Morrison}

Department of Microbiology, Montana State University, Bozeman, Montana, USA

\begin{abstract}
Tissue tropism of clinical ocular and genital Chlamydia trachomatis strains is shown to be linked to the tryptophan synthase genotype (see related article beginning on page 1757). It is suggested that, in the presence of IFN- $\gamma$, which depletes available tryptophan, there exist unique host-parasite interactions that may contribute to persistent chlamydial infection.
\end{abstract}

J. Clin. Invest. 111:1647-1649 (2003). doi:10.1172/JCI200318770.

Chlamydial infections are noted for the broad array of clinically distinct manifestations that they produce, ranging from acute self-limiting ocular and genital infections to chronic inflammatory diseases that result in blindness or infertility. Trachoma, an ocular

Address correspondence to: Richard P. Morrison, Department of Microbiology, Lewis Hall, Room 109, Montana State University, Bozeman, Montana 59717, USA. Phone: (406) 994-7959; Fax: (406) 994-4926; E-mail: morrison@montana.edu.

Conflict of interest: The author has declared that no conflict of interest exists.

Nonstandard abbreviations used: elementary body (EB); reticulate body (RB); indoleamine 2,3-dioxygenase (IDO). infection, is caused by Chlamydia trachomatis serovars A-C and is a primary cause of preventable blindness. The majority of C. trachomatis serovars (D-K) cause urogenital infection, which can progress to serious genital tract disease in men and women. A genetic basis for the remarkable tissue tropism of the various chlamydial serovars (i.e., ocular vs. urogenital strains) was first proposed by FehlnerGardiner et al., based upon their analysis of the tryptophan synthase gene cluster (trpRBA) of laboratory strains of chlamydiae (1). They found that the tryptophan synthase genotype is closely linked to the tissue tropism of ocular and genital chlamydial strains; urogenital serovars possess a functional tryptophan synthase and are capable of utilizing indole as a substrate for tryptophan synthesis, whereas ocular isolates possess a nonfunctional tryptophan synthase. In this issue of the JCI, Caldwell et al. have extended that earlier work to include the characterization of tryptophan synthase genes from hundreds of clinical isolates, thereby confirming the correlation between chlamydial tryptophan synthase genotype and tissue tropism (2).

Between the extremes of acute selflimiting infection and chronic inflammatory disease lies the notion of persistent and/or chronic states of chlamydial infection. Cell culture systems have been used for a number of years to demonstrate and study chlamydial persistence in vitro (3). However, the experimental documentation of persistent infection in the human host and of the mechanisms of in vivo persistence has been a much greater challenge. A particularly intriguing hypothesis put forth by the authors is that tryptophan synthase genes function as chlamydial virulence factors and may be involved in the maintenance of persistent/chronic chlamydial infection. That hypoth- 


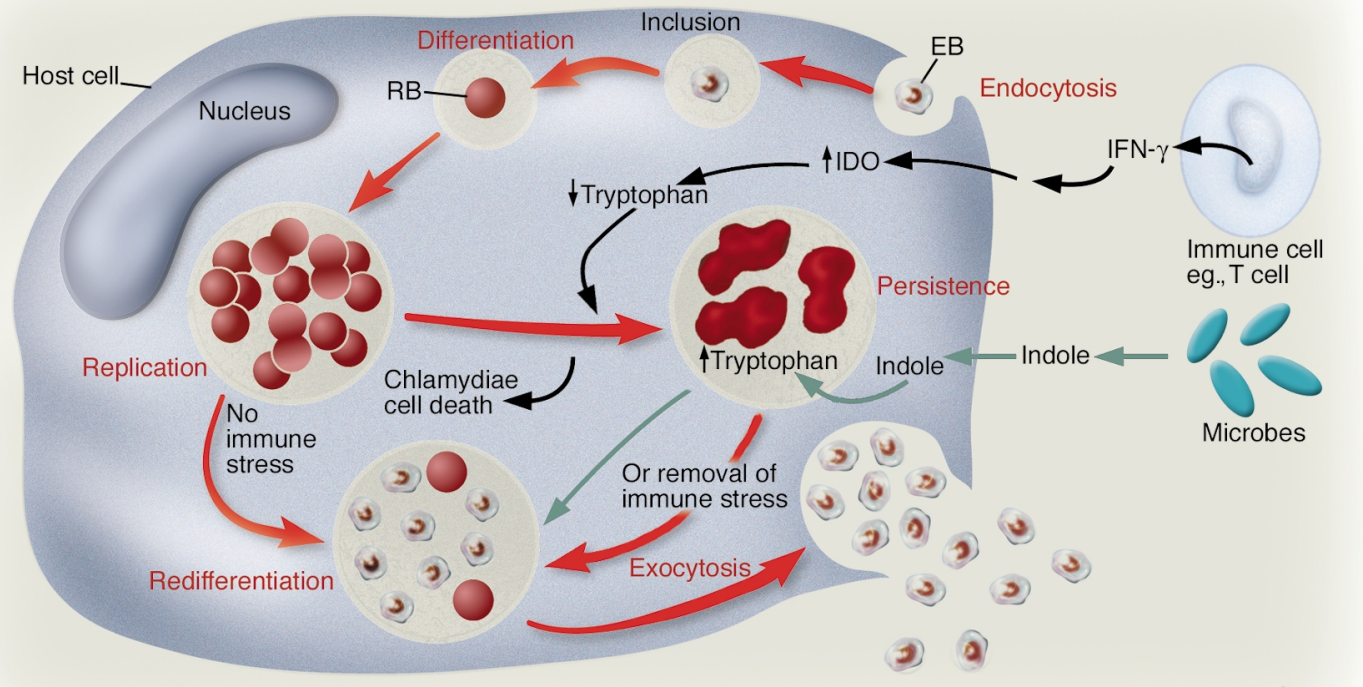

by Ken Beauchamp J. Clin. Invest

\begin{abstract}
Figure 1
Chlamydial infection is initiated by the attachment of the infectious EB to the host cell, followed by entry of the EB into a membrane-bound vesicle, termed an inclusion. The inclusion evades fusion with host lysosomes, and the EB rapidly differentiates into an RB that replicates by binary fission within the inclusion. Following several rounds of replication, the RBs reorganize and form infectious EBs, which are released from the cell. Under certain conditions, such as in an inflammatory environment where IFN- $\gamma$ is produced, the intracellular growth of ocular and urogenital chlamydial strains may be altered. IFN- $\gamma$ induces cellular IDO, which results in a marked decrease in available tryptophan. Depletion of tryptophan either results in chlamydiae cell death or causes chlamydiae to adopt a noninfectious, nonreplicating form that retains viability (persistence). The outcome is dependent on the level and duration of tryptophan depletion and the tryptophan synthase genotype of the infecting chlamydial strain. Persistent forms of chlamydiae can redifferentiate into infectious EBs upon removal of IFN- $\gamma$ and subsequent replenishing of intracellular tryptophan pools. Alternatively, even in an IFN- $\gamma$-rich environment, strains of chlamydiae that possess a functional tryptophan synthase (i.e., genital strains) may use indole (perhaps produced by local microbial flora) as a substrate for tryptophan synthesis to counter the growth inhibitory effects of IFN- $\gamma$. This model is far from complete, and the biological processes involved are likely much more complex and interrelated than depicted here. However rudimentary, though, the proposed model provides a reasonable representation of our current understanding.
\end{abstract}

esis is particularly relevant when one considers the key role played by IFN- $\gamma$ in host defense against chlamydial infection. Caldwell et al. propose a mechanism by which genital chlamydial strains may counter the growth inhibitory effects of IFN- $\gamma$ by utilizing indole produced by vaginal microbial flora as a substrate for tryptophan synthesis, thus allowing for the continued survival of chlamydiae within a growth-inhibiting environment (2).

\section{The intracellular developmental cycle of Chlamydia}

Key to understanding the pathophysiology of chlamydial disease is an appreciation of the chlamydial developmental cycle (Figure 1) (4). Chlamydial infection is initiated by the attachment to a susceptible host cell of the infectious, metabolically inert elementary body (EB), which then enters the cell within a membrane-bound vesicle, termed an inclusion. The EB rapidly differentiates into a reticulate body (RB), which replicates by binary fission within the confines of the inclusion. Following several rounds of replication, the RBs reorganize to form infectious EBs, which are released by cytolysis from the cell to initiate new infections. Deviations from this typical developmental cycle have been experimentally induced by a variety of stimuli, including IFN- $\gamma$, antibiotics, and nutrient deprivation (3). Pertinent to the current discussion is the ability of these stimuli, particularly IFN- $\gamma$, to alter chlamydial growth and facilitate persistent or chronic infection.

\section{Experimental persistence in cell culture}

It has long been known that IFN- $\gamma$ alters the growth of $C$. trachomatis in cell culture systems (5). Chlamydial serovars and species display differen- tial susceptibilities to the inhibitory effects of IFN- $\gamma$, which range from minimal to marked inhibition of chlamydial growth (6). In addition, IFN- $\gamma$ induces persistent chlamydial infections in cell culture systems that are characterized by viable, but noncultivatable, chlamydiae, which display aberrant morphology $(7,8)$. These aberrant forms of chlamydiae remain viable for over one month in cell culture and differentiate into infectious EBs when IFN- $\gamma$ is removed from the cell culture system $(9,10)$.

In theory, the mechanisms by which IFN- $\gamma$ could inhibit chlamydial growth are many, due to the pleomorphic effects of IFN- $\gamma$ on host cell function. However, one mechanism, which is particularly germane to the current discussion, is the induction by IFN- $\gamma$ of the tryptophan-decyclizing enzyme indoleamine 2,3-dioxygenase (IDO). Depletion of intracellular pools of 
tryptophan by IDO essentially starves chlamydiae of this essential amino acid, rendering them incapable of differentiating into infectious EBs (Figure 1) $(10,11)$. As noted by Caldwell et al., the resistance pattern of the various chlamydial strains to the inhibitory effects of IFN- $\gamma$ correlates to polymorphisms in tryptophan synthase genes (2). Thus, ocular serovars, which have a nonfunctional tryptophan synthase and are unable to synthesize tryptophan, are more sensitive to IFN- $\gamma$ mediated inhibition than genital serovars, which have a functional tryptophan synthase and are capable of utilizing indole as a substrate for tryptophan synthesis.

\section{Clinical persistence}

Persistence of chlamydial infection in the human host is at best incompletely defined $(12,13)$. Chlamydial infections are known to be particularly insidious, and chronic inflammatory conditions are a frequent consequence of untreated chlamydial infection. Because chlamydiae respond to a variety of environmental stimuli that alter their growth characteristics (e.g., IFN- $\gamma$ ), there exists the potential for chlamydiae to establish a chronic or persistent relationship with the host. If persistent or chronic infections are established, then those infections may serve as a reservoir for new infections, contribute to the immunopathological consequences of infection, or require alternative therapeutic approaches. Thus, understanding the consequences of persistent chlamydial infection could be important to the control and prevention of chlamydial disease.

IFN- $\gamma$ is an important mediator of protective immunity to chlamydial infection (14). Consequently, the possibility that chlamydiae may grow in an environment rich in IFN- $\gamma$ and the potential for chlamydiae to establish persistent or chronic infections in the presence of IFN- $\gamma$ are two infection outcomes that could have important clinical implications. Caldwell et al. discuss quite eloquently how genital strains of chlamydiae could utilize indole produced by vaginal microbes to survive in an IFN- $\gamma$-rich, tryptophan-limiting environment (Figure 1) and how survival under such conditions might facilitate the development of chronic infections (2). Moreover, if the in vitro observation of IFN- $\gamma-$ mediated persistent infection holds for human infection, then perhaps in an IFN- $\gamma$-rich environment, chlamydiae could persist as morphologically aberrant, nonculturable forms. If so, would those persistent chlamydiae be susceptible to therapeutic doses of antimicrobials typically prescribed to treat infection? Although apparent treatment failures have been reported, there is no direct in vivo evidence to support the notion that those failures resulted from persistent chlamydiae being more refractory to antimicrobial chemotherapy. However, recent studies using an in vitro cell culture system suggest that persistent forms of chlamydiae are more refractory to antimicrobial therapy than chlamydiae grown under normal cell culture conditions (P. Wyrick, personal communication).

Much remains to be learned about the pathogenesis of chlamydial disease, but the study by Caldwell et al. represents a seminal contribution to our understanding of chlamydiae-host interactions (2). Further characterization of the polymicrobial environment of the female genital tract during chlamydial infection and the development of experimental animal models of defined polymicrobial infections will be needed to assess the full impact of tryptophan synthase gene polymorphisms on the pathogenesis of chlamydial disease. Additional studies will also be needed to more fully understand why ocular strains have lost functional tryptophan synthase activity even though they face the same IFN- $\gamma$ selection pressure as genital strains and to define what other biological differences may exist between ocular and genital strains that might contribute to tissue tropism and disease pathogenesis. Investigat- ing the interplay between chlamydiae and its environment will impart a more thorough understanding of host-microbial interactions and may provide important insight into novel approaches for the treatment and prevention of chlamydial disease.

\section{Acknowledgments}

The author acknowledges grant support from the National Institutes of Health (AI-038991) and the American Heart Association (0150034N).

1. Fehlner-Gardiner, C., et al. 2002. Molecular basis defining human Chlamydia trachomatis tissue tropism: a possible role for tryptophan synthase. J. Biol. Chem. 277:26893-26903.

2. Caldwell, H.D., et al. 2003. Polymorphisms in Chlamydia trachomatis tryptophan synthase genes differentiate between genital and ocular isolates. J. Clin. Invest. 111:1757-1769. doi:10.1172/JCI200317793.

3. Beatty, W.L., Morrison, R.P., and Byrne, G.I. 1994. Persistent chlamydiae: from cell culture to a paradigm for chlamydial pathogenesis. Microbiol. Rev. 58:686-699.

4. Hackstadt, T. 1999. Cell biology. In Chlamydia: intracellular biology, pathogenesis, and immunity. R.S Stephens, editor. American Society for Microbiology Press. Washington, D.C., USA. 101-138.

5. Kazar, J., Gillmore, J.D., and Gordon, F.B. 1971. Effect of interferon and interferon inducers on infections with a nonviral intracellular microorganism, Chlamydia trachomatis. Infect. Immun. 3:825-832.

6. Morrison, R.P. 2000. Differential sensitivities of Chlamydia trachomatis strains to inhibitory effects of gamma interferon. Infect. Immun. 68:6038-6040.

7. Beatty, W.L., Byrne, G.I., and Morrison, R.P. 1993 Morphologic and antigenic characterization of interferon-gamma mediated persistent Chlamydia trachomatis infection in vitro. Proc. Natl. Acad. Sci. U. S. A. 90:3998-4002.

8. Beatty, W.L., Morrison, R.P., and Byrne, G.I. 1994 Immunoelectron-microscopic quantitation of differential levels of chlamydial proteins in a cell culture model of persistent Chlamydia trachomatis infection. Infect. Immun. 62:4059-4062.

9. Beatty, W.L., Morrison, R.P., and Byrne, G.I. 1995. Reactivation of persistent Chlamydia trachomatis infection in cell culture. Infect. Immun. 63:199-205.

10. Beatty, W.L., Belanger, T.A., Desai, A.A., Morrison, R.P., and Byrne, G.I. 1994. Tryptophan depletion as a mechanism of gamma interferon-mediated chlamydial persistent. Infect. Immun. 62:3705-3711.

11. Byrne, G.I., Lehmann, L.K., and Landry, G.J. 1986. Induction of tryptophan catabolism is the mechanism for gamma-interferon-mediated inhibition of intracellular Chlamydia psittaci replication in T24 cells. Infect. Immun. 53:347-351.

12. Dean, D., Suchland, R.J., and Stamm, W.E. 2000. Evidence for long-term cervical persistence of Chlamydia trachomatis by opm 1 genotyping. J. Infect. Dis. 182:909-916.

13. Campbell, L.A., et al. 1993. Detection of Chlamydia trachomatis deoxyribonucleic acid in women with tubal infertility. Fertil. Steril. 59:45-50.

14. Morrison, R.P., and Caldwell, H.D. 2002. Immunity to murine chlamydial genital infection. Infect. Immun. 70:2741-2751. 